\title{
Mobility Experiments with Microrobots for Minimally Invasive Intraocular Surgery
}

\author{
Franziska Ullrich ${ }^{1}$, Christos Bergeles ${ }^{1,2}$, Juho Pokki ${ }^{1}$, Olgac Ergeneman ${ }^{1}$, Sandro Erni ${ }^{1}$, \\ George Chatzipirpiridis ${ }^{1}$, Salvador Pané ${ }^{1}$, Carsten Framme ${ }^{3,4}$, Bradley J. Nelson ${ }^{1}$ \\ ${ }^{1}$ Institute of Robotics and Intelligent Systems, ETH Zurich, 8092 Zurich, Switzerland \\ ${ }^{2}$ Department of Cardiovascular Surgery, Boston Children's Hospital, Harvard Medical School, \\ Boston, 02115, Massachusetts, USA \\ ${ }^{3}$ Inselspital, Universitätsspital Bern, 3010 Bern, Switzerland \\ ${ }^{4}$ Hannover Medical School (MHH), 30625 Hannover, Germany
}

Correspondence to:

Bradley J. Nelson, Institute of Robotics and Intelligent Systems, ETH Zurich

CLA H15.2, Tannenstrasse 3, 8092 Zurich, Switzerland

bnelson@ethz.ch

Word Count: 4096 


\section{Abstract}

Purpose. To investigate microrobots as an assistive tool for minimally invasive intraocular surgery and to demonstrate mobility and controllability inside the living rabbit eye.

Methods. A system for wireless magnetic control of untethered microrobots was developed. Mobility and controllability of a microrobot are examined in different media, specifically vitreous, balanced salt solution (BSS) and silicone oil. This is demonstrated through ex vivo and in vivo animal experiments.

Results. The developed electromagnetic system enables precise control of magnetic microrobots over a workspace that covers the posterior eye segment. The system allows for rotation and translation of the microrobot in different media (vitreous, BSS, silicone oil) inside the eye.

Conclusion. Intravitreal introduction of untethered mobile microrobots can enable suture-less and precise ophthalmic procedures. Ex vivo and in vivo experiments demonstrate that microrobots can be manipulated inside the eye. Potential applications are targeted drug-delivery for maculopathies such as age-related macular degeneration (AMD), intravenous deployment of anti-coagulation agents for retinalvein occlusion (RVO) and mechanical applications, such as manipulation of epiretinal membrane peeling (ERM). The technology has the potential to reduce the invasiveness of ophthalmic surgery and assist in the treatment of a variety of ophthalmic diseases.

Keywords: Microrobot, intravitreal, minimally invasive surgery, magnetic, in vivo, vitreous, wireless, untethered 


\section{Introduction}

With the advent of modern ophthalmic devices such as $23 \mathrm{G}$ and $25 \mathrm{G}$ tools, ophthalmology is moving towards more minimally invasive surgery, resulting in less inflammation, decreased operating times and less damage to the conjunctiva ${ }^{1}$. In order to further the advantages of minimally invasive ophthalmic surgery, several researchers are developing smart miniature tools or robot-assisted devices that assist in ocular surgery and help overcome the limits of human performance due to their high precision ${ }^{2,3,4,5,6}$. Here we describe a microrobotic approach for minimally invasive surgery in the posterior eye segment as a future treatment for various ocular impairments and present results of in vivo mobility experiments of wirelessly controlled devices $285 \mu \mathrm{m}$ diameter and $1800 \mu \mathrm{m}$ length in lapine eyes. The small devices that we call "microrobots" are injected into the vitreous cavity through a small incision in the pars plana region of the sclera and can be guided wirelessly to the pathological site where they are operated by a surgeon to perform a required treatment such as mechanical manipulation or targeted drug delivery.

The first use of a device of this type will be for directing intravitreal inserts near pathological sites on the retina in order to reduce the volume of drug required for treatment, thus extending the length of time for which the insert can provide a therapeutically relevant dose. While topical administration is most commonly used to treat ocular disorders, the physiological barriers to topical absorption are considerable. High administration frequency and high drug doses are required ${ }^{7}$, because only a small fraction (approximately 1 to 10\%) of the topically applied drug permeates the eye $^{8}$. Therefore, macular diseases are frequently administered by intravitreal injections, predominantly using anti-VEGF (vascular endothelial growth factor) agents ${ }^{9}$. Intravitreal administration is superior to topical administration and systemic drug delivery, where toxic side effects to non-targeted tissue can occur due to high doses ${ }^{7,10}$.

Intravitreal inserts are used for single injection, long-term drug delivery. These devices can be loaded with a variety of drugs targeting a broad range of ocular diseases. A pellet device comprising a silicone shell has been developed to release therapeutic intraocular doses of thalidomide and could be used for the treatment of subretinal neovascularization ${ }^{11}$. Several non-bioerodible inserts have been suggested, which are loaded with ocular corticosteroid fluocinolone acetonide for the treatment of chronic, non-infectious uveitis, and have exhibited stable drug release over a period of approximately three months ${ }^{11}$. Ozurdex ${ }^{\mathrm{TM}}$, a biodegradable intravitreal implant, is approved as a first-line treatment for $\mathrm{RVO}^{12}$. These ocular inserts lack the ability to move and, therefore, cannot be safely directed to a pathological site within the eye. Additionally, the removal of some of these devices requires a vitrectomy, which bears risks of complications. The addition of mobility to intravitreal devices to guide them to the diseased area will allow much slower release drug delivery while providing an equivalent dosage to the pathology due to the nonlinear nature of Fick's law of diffusion. 
In addition to guiding intravitreal inserts, other mechanical operations inside the vitreous cavity may also be performed in the future. Pars plana vitrectomy can successfully treat retinal detachments, macular-hole formation, vitreo-macular traction syndromes, retinal vein occlusion (RVO), proliferative DR, vitreous hemorrhages, and diabetic macular edema (DME) by epiretinal membrane (ERM) peeling ${ }^{13}$. The delicate structure of the retina is at risk during surgery due to a lack of tactile information arising from the limits of human force perception ${ }^{14,15}$. Ophthalmic surgery is mechanically difficult and may lead to complications such as cataract, vitreous cavity hemorrhage or retinal detachment ${ }^{16,17}$.

Microrobots are a new wireless tool for future suture-less ocular surgery and are rapidly gaining interest as in vivo diagnostic and therapeutic devices ${ }^{18,19,20}$. In order to utilize these microrobots in future ophthalmic applications such as ERM, localized drug delivery or puncturing retinal veins, they must exhibit biocompatibility, mechanical stability, hypoallergenic, noncarcinogenic, and chemically inert properties ${ }^{11}$. Furthermore, the technology must allow the surgeon to precisely control rotation and translation of the microrobot inside the vitreous. This work investigates the mobility of intraocular microrobots as potential tools for microsurgery. The aim is to understand robot mobility in the posterior eye segment in vitreous humor as well as after replacement of the vitreous with different media. This paper utilizes an electromagnetic system capable of dexterous micromanipulation of steerable magnetic intraocular microrobots. Mobility and control of these microrobots are successfully demonstrated in vivo. Microrobots can be removed in a controllable and minimally invasive manner, potentially avoiding a vitrectomy, and, thus making ophthalmic surgical interventions accessible to a larger set of ophthalmic surgeons and more acceptable by patients.

\section{Materials and Methods}

\subsection{Intraocular Microrobot}

Figure 1(a) shows a microrobot, which is injected, into the posterior section of the eye through the pars plana region of the sclera. The microrobot is wirelessly controlled and can be removed by a magnetic tool. The soft-magnetic microrobot has the shape of a hollow cylinder with outer diameter of $285 \mu \mathrm{m}$ and inner diameter of $125 \mu \mathrm{m}$; its length is $1800 \mu \mathrm{m}$. The outer diameter is chosen such that the microrobot fits a $23 \mathrm{G}$ needle, as shown in Figure 1(b). The microrobot is rendered non-toxic using polypyrrole or inert metallic coatings, which have been tested for cell viability by Sivaraman et al. ${ }^{\mathbf{2 1}}$ and customized fabrication methods allow for high flexibility in diameter, length and magnetic volume $^{21,22}$. 


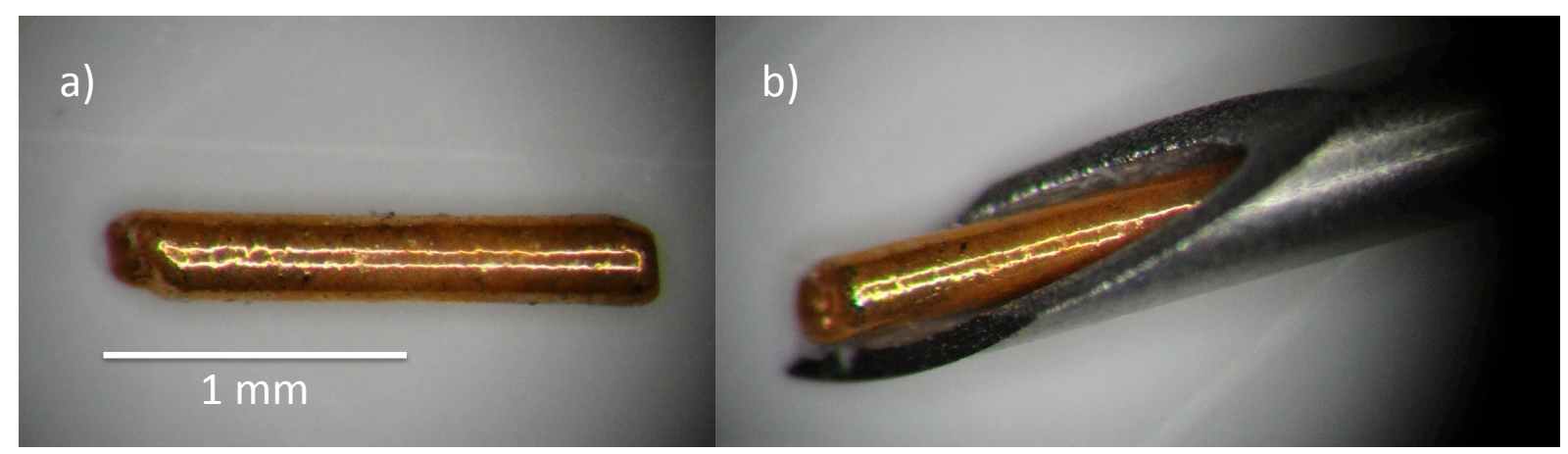

Figure 1: (a) Cylindrical microrobot with outer diameter of $285 \mu \mathrm{m}$, inner diameter of $125 \mu \mathrm{m}$ and length of $1800 \mu \mathrm{m}$. (b) A microrobot in a $23 \mathrm{G}$ needle

\subsection{OctoMag: An Electromagnetic System for Magnetic Microrobot Control}

The OctoMag, an electromagnetic system that allows for unrestrained wireless electromagnetic control, was introduced by Kummer et al. ${ }^{23}$. The OctoMag is capable of controlling magnetic devices in three dimensions (3D). The workspace of this magnetic system is approximately $20 \mathrm{~mm} \times 20 \mathrm{~mm} \times 20 \mathrm{~mm}$ and covers the posterior segment of the human eye, which has a an approximate diameter of $20 \mathrm{~mm}^{24,25}$. The OctoMag consists of eight electromagnets arranged in a hemispherical configuration. The electromagnets are operated with direct currents and are equipped with soft-magnetic cores, and can generate an electromagnetic field of up to $40 \mathrm{mT}$ with gradients up to $1 \mathrm{~T} / \mathrm{m}$. The rotation of a microrobot is controlled by the orientation of the applied magnetic field, whereas the magnitude of the force applied to the microrobot is a function of the field gradient. The current system, shown in Figure 2, can accommodate a small animal (e.g. rabbit) for in vivo experiments.

In ex vivo experiments a Leica M80 stereomicroscope [Leica Microsystems, Switzerland] equipped with a Grasshopper 03K2C-C camera [Pointgrey, British Columbia, Canada] and a wide field enhanced BIOM lens [WFE 53602, Oculus, Germany] for non-contact $120^{\circ}$ wide-angle observation views the workspace from above. The microscope has an objective lens $(f=200 \mathrm{~mm})$ from the Leica retinal surgery series. For in vivo experiments a Leica Wild series microscope equipped with the same Grasshopper camera is used to observe the workspace from above. To minimize the reflections from the optical interface a disposable planoconcave vitrectomy contact lens [S5-7010u, FCI Ophthalmics, USA] is placed on the eye. 


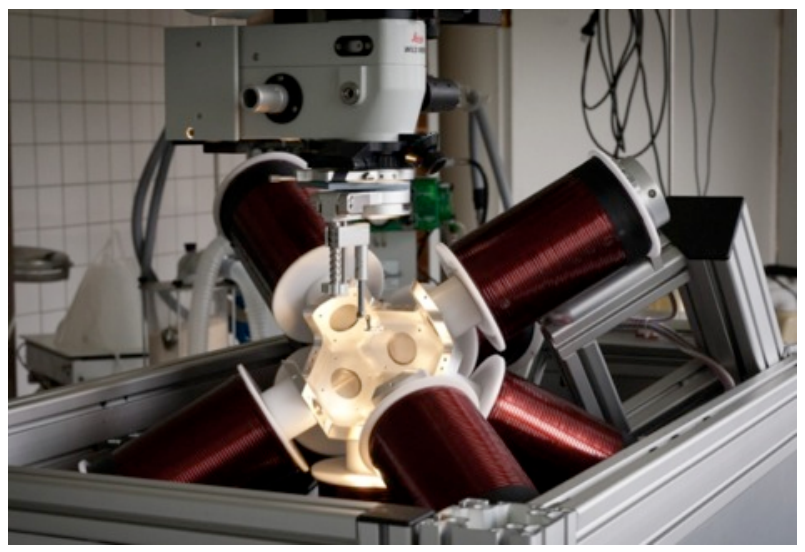

Figure 2: OctoMag, utilizing eight electromagnets for wireless magnetic microrobot control. A surgical microscope and a BIOM allow for observation from above.

\subsection{Porcine Eyes (ex vivo)}

It has been suggested that the viscoelastic behavior of the central vitreous region of a pig closely resembles that of the central vitreous region of a human eye in terms of rheological parameters ${ }^{26}$. The same study reports that the anterior and posterior regions of the porcine vitreous resemble a dense gel, whereas the human vitreous has a thinner and in some cases aqueous consistency. Similarly, it has been observed, that human vitreous undergoes liquefaction with increasing age ${ }^{27}$. It can be concluded that, with respect to the properties of the vitreous humor, porcine eyes act as a stiff model of the human eye.

In order to account for the rapid loss of hyaluronic acid of the vitreous upon removal from the eye globe and to sustain the intraocular pressure (IOP), microrobotic mobility experiments are conducted inside the eye globe without destroying the natural structure of the eye. Ex vivo experiments in porcine cadaver eyes are performed one hour post mortem.

\subsection{Lapine Eyes (in vivo)}

In order to evaluate the ability of intravitreal microrobots to move in the living eye, in vivo mobility experiments were conducted in rabbits. Due to their characteristic bright red eyes, the New Zealand white rabbit breed ( 9 month-old females) was chosen for this study. Experiments are undertaken in five individual rabbit eyes, three of which are vitrectomized. One eye is subsequently filled with BSS and two with silicone oil (AK 1000). The rabbit is anesthetized and its head is placed inside the workspace of the OctoMag, such that the studied eye is located in its center, as illustrated in Figure 3.

The protocols concerning animal housing, treatment and monitoring were approved by the Swiss Veterinary Office according to the Swiss decree on animal protection ${ }^{28}$ and adhere to the ARVO guidelines for animal experiments. This study was undertaken in cooperation with ophthalmologists from the University Hospital Bern and the Veterinary Hospital Zurich. 


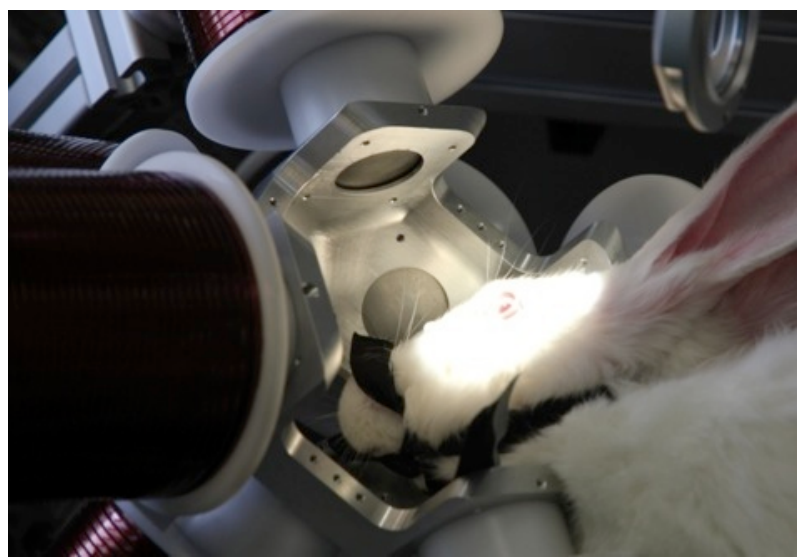

Figure 3: Anesthetized rabbit placed with the studied eye centrally in the OctoMag workspace

\subsection{Mobility Experiments}

A microrobot is injected into the central vitreous humor of the porcine or lapine eyes with a syringe equipped with a $23 \mathrm{G}$ needle. After insertion of the microrobot, the eye is placed centrally in the workspace of the OctoMag for experimentation. After experimentation the microdevice is removed from the vitreous cavity using a magnetic tool that is manufactured from a standard syringe. A magnetic wire is inserted into the needle, which attracts the microrobot if a low magnetic field is applied that also orients the device. Subsequently, the microrobot can be pulled into the needle and removed from the eye. Injection and removal of an intraocular microrobot are illustrated in Figure 4.

a)

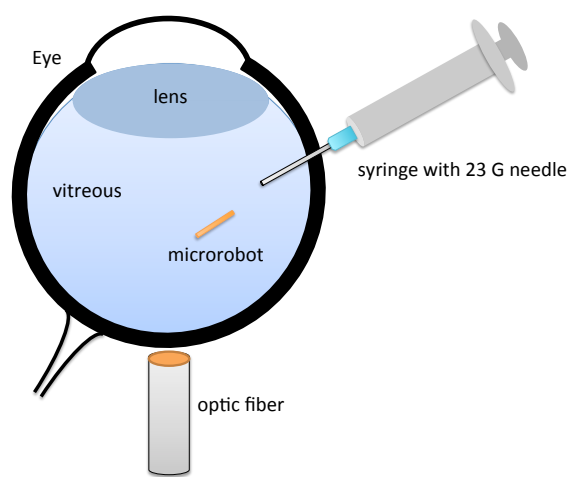

b)

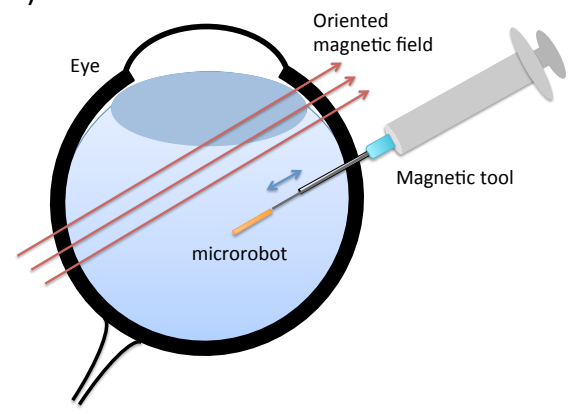

Figure 4: a) Schematic of an eye with transscleral illumination and injected robot. b) Removal of intravitreal microrobot using a magnetic tool. A weak magnetic field is applied to orient the microrobot.

\subsubsection{Rotational Mobility}

The intraocular microrobot is rotated in the plane, normal to the line of vision in the posterior region of the eye. The rotation of the microrobot is caused by the rotation of the applied magnetic field, 
which is generated by the OctoMag. The microrobot rotational mobility is examined in lapine vitreous, BSS and silicone oil in vivo and in porcine vitreous ex vivo.

In order to evaluate the rotational mobility of an untethered intraocular microrobot and its response to magnetic field strength, the field magnitude is set to $10 \mathrm{mT}, 20 \mathrm{mT}, 30 \mathrm{mT}$ and $40 \mathrm{mT}$ whereas the field's rotational frequency is held constant at $1 \mathrm{~Hz}$ and the intraocular microrobot mobility is recorded. In a second series of experiments, the rotation of an intraocular microrobot is investigated at constant field magnitude whereas the field rotation frequency is set to $0.05 \mathrm{~Hz}, 0.1 \mathrm{~Hz}$, $0.5 \mathrm{~Hz}, 1 \mathrm{~Hz}$ and $2 \mathrm{~Hz}$. For experiments in vitreous and silicone oil the field magnitude is held constant at $30 \mathrm{mT}$. It is set to a lower field magnitude $(10 \mathrm{mT})$ for experiments in BSS due to an unstable response of the microrobot in BSS at higher field strengths.

\subsubsection{Translational Mobility}

In order to examine the translational mobility of the microrobot inside the eye globe, a magnetic gradient is applied. The microrobot is surrounded by lapine vitreous, BSS or silicone oil in in vivo experiments and porcine vitreous in ex vivo testing. The magnetic field gradient is increased from 0 to $500 \mathrm{mT} / \mathrm{m}$, resulting in translational movement of the intraocular microrobot whereas the field magnitude and orientation are held constant for each experiment. Experiments are undertaken for magnetic field magnitudes of $10 \mathrm{mT}, 20 \mathrm{mT}, 30 \mathrm{mT}$ and $40 \mathrm{mT}$. Furthermore, the translational mobility of the microdevice in porcine and lapine vitreous is compared.

\subsubsection{Motion Tracking}

The microscope equipped with a camera takes consecutive images of the inside of the eye globe with a speed of $15 \mathrm{~Hz}$. In order to track the microrobot movement a script is written in MATLAB, which returns the robot orientation and position in every image. Due to minor movement of the rabbit eye during in vivo experiments, noise occurs and the microrobot position data must be filtered using a moving average filter with window size of ten data points for further analysis.

\section{Results and Discussion}

\subsection{Rotational Movement}

As a result of the rotating magnetic field, the intraocular microrobot follows the field with a time delay. Figure 5 a) indicates the angle of rotation of the magnetic field, $\alpha_{\text {field }}$, and the angle of rotation of the microrobot, $\alpha_{\text {robot }}$. The response of the robot to a $10 \mathrm{mT}$ field rotating counter clockwise with frequency of $0.5 \mathrm{~Hz}$ is illustrated in Figure $5 \mathrm{~b}$ ). A black arrow indicates the orientation of the magnetic field. 

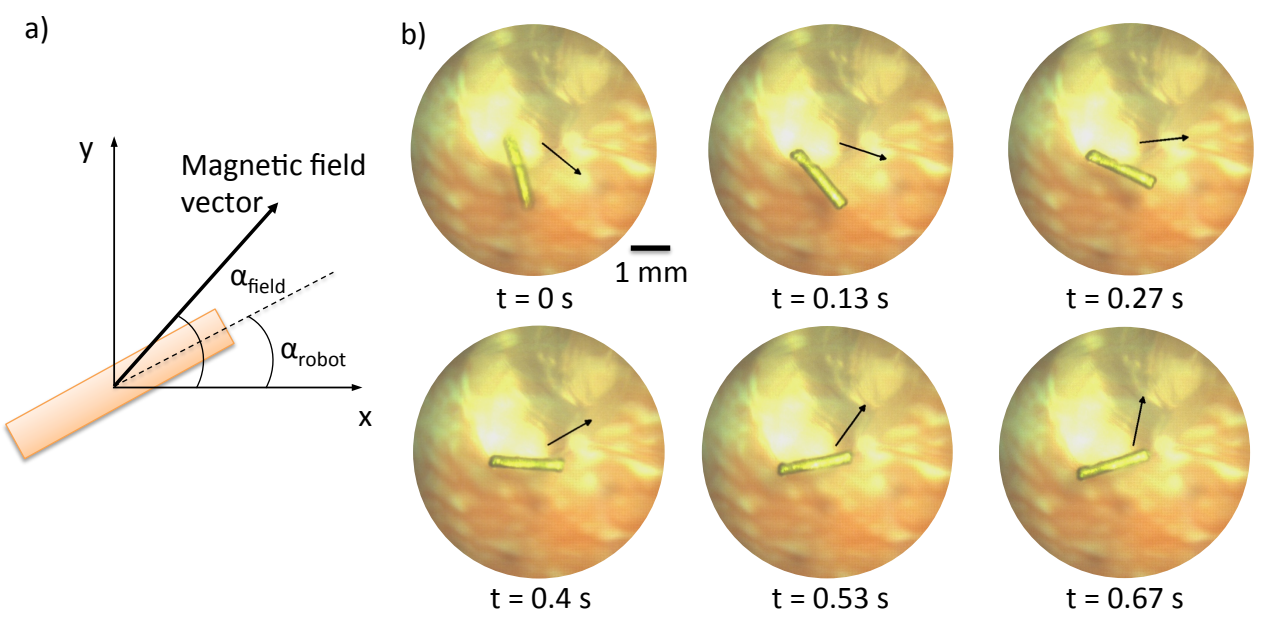

Figure 5: a) Inertial coordinate frame, where the $x-y$ plane is perpendicular to the line of vision. b) A goldcoated intravitreal microrobot inside the vitreous of a live rabbit. As the applied magnetic field (indicated by a black arrow) rotates anti-clockwise, the microrobot follows with a time delay.

\subsubsection{Constant Magnetic Field Frequency}

Figure 6 graphs the angle of rotation of an applied magnetic field at various field magnitudes, rotating at $1 \mathrm{~Hz}$, and the rotation of a microrobot inside the eye over time, where $\mathrm{T}$ is the period of rotation in seconds $(\mathrm{T}=1 \mathrm{~s})$. Figure 6 a) illustrates microrobot rotation in lapine vitreous (in vivo), subfigures $\mathrm{b}$ ) and c) show the microrobot movement in BSS and silicone oil (in vivo), respectively. Figure $6 \mathrm{~d}$ ) shows the rotational motion of the microrobot in porcine vitreous (ex vivo). The black arrow indicates the time delay between the rotating field and the microrobot rotation.

It is observed that the plotted angles of rotation show almost no change for different field magnitudes in all four surrounding media. The time delay of the microrobot rotation compared to the field rotation is similar for all recorded field magnitudes at constant frequency in each medium. Therefore, it is concluded that the magnitude of the rotating magnetic field has no influence on the time delay of the microrobot. Unlike in vitreous, the microrobot shows very unstable movement in BSS at $40 \mathrm{mT}$ (not plotted). This instability of the microrobot can be explained with an increasing magnetic drift inside the OctoMag workspace due to the increasing field strength. Furthermore, the viscosity of BSS is much lower than that of vitreous, thus, less damping of the movement is observed and instabilities are less controllable. In porcine vitreous the microrobot movement is hindered at field strength of $10 \mathrm{mT}$ (not plotted), presumably due to collagen fibers inside the vitreous. 

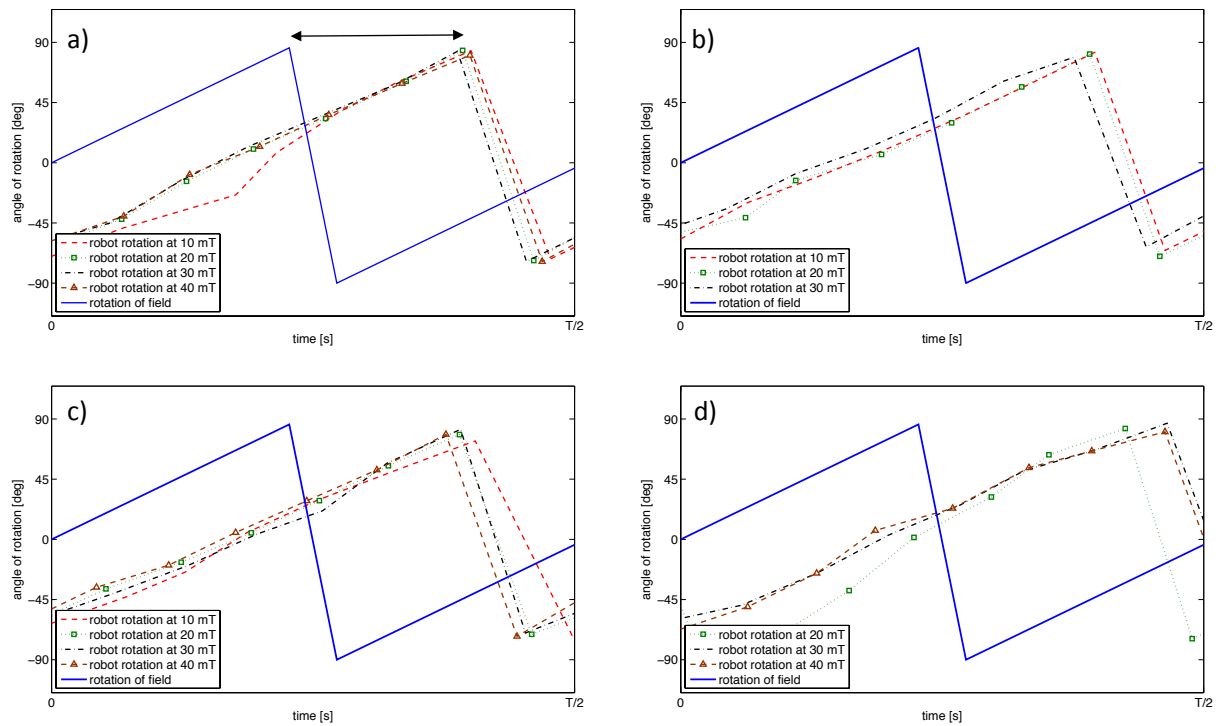

Figure 6: Angle of rotation of the applied magnetic field (rotation frequency $1 \mathrm{~Hz}$ ) and the microrobot a) in lapine vitreous (in vivo), b) inside the lapine eye in BSS (in vivo), c) inside the lapine eye in silicone oil (in vivo), d) porcine vitreous (ex vivo) at field magnitudes $10 \mathrm{mT}, 20 \mathrm{mT}, 30 \mathrm{mT}, 40 \mathrm{mT}$

\subsubsection{Constant Magnetic Field Magnitude}

Figure 7 illustrates the angle of rotation of the rotating magnetic field and the intraocular microrobot surrounded by a) lapine vitreous, b) BSS, c) silicone oil and d) porcine vitreous. The rotation is plotted against time, where $\mathrm{T}$ is the period of rotation at a corresponding frequency. It is observed, that the relative time delay between the field angle of rotation and the microrobot rotation increases with decreasing period $\mathrm{T}$, and thus increasing field frequency. It is concluded that the time delay between robot rotation and the rotating magnetic field corresponds to rotational field frequency. Furthermore, it is observed that the time delay between angle of rotation of the field and the microrobot is generally larger in vitreous (lapine and porcine) than in BSS and silicone oil. This observation is attributed to the presence of elastic collagen fibers in vitreous. 

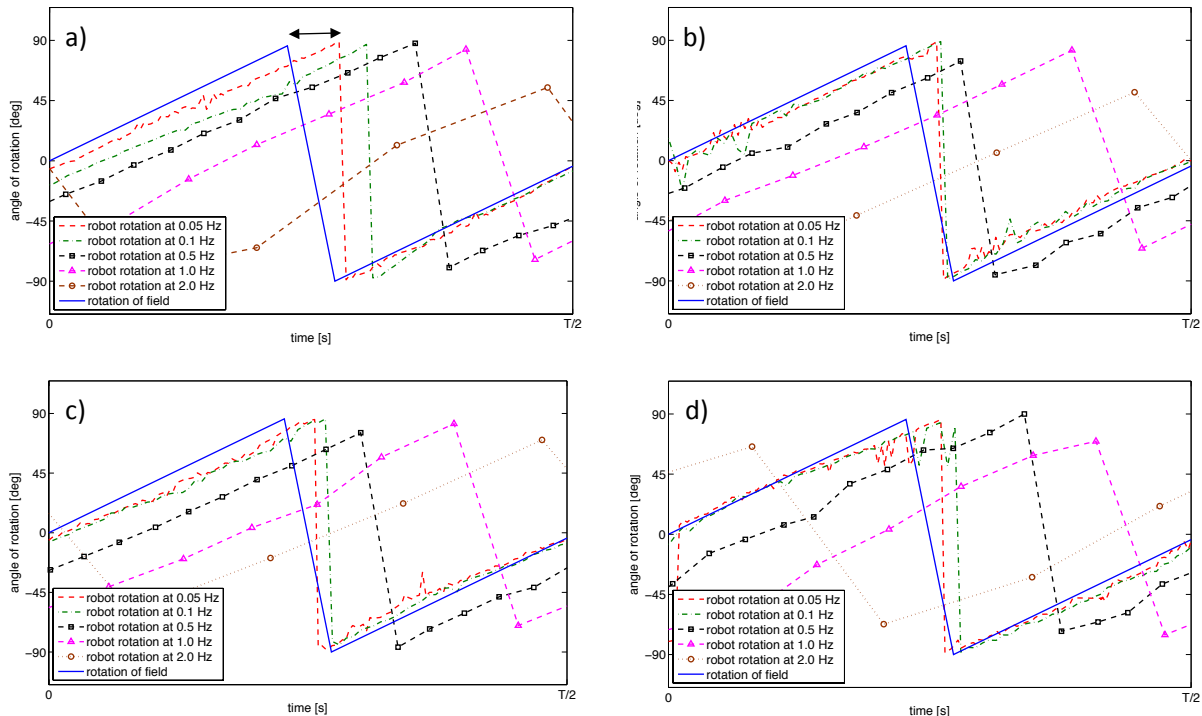

Figure 7: Angle of rotation of the applied magnetic field and the microrobot at frequencies $0.05 \mathrm{~Hz}$, $0.1 \mathrm{~Hz}, 0.5 \mathrm{~Hz}, 1 \mathrm{~Hz}, 2 \mathrm{~Hz}$, in a) lapine vitreous (in vivo; $30 \mathrm{mT}$ ), b) BSS (in vivo $10 \mathrm{mT}$ ) c) silicone oil (in vivo; $30 \mathrm{mT}), \mathrm{d})$ porcine vitreous (ex vivo; $30 \mathrm{mT}$ )

As suggested in literature vitreous is not only a viscous fluid, but also has elastic properties ${ }^{29}$, which are observed in some experiments described in this work. Figure 8 shows the corresponding plots for such a case, where the microrobot is unable to accomplish a full rotation due to being caught in collagen fibers. The figure shows an applied magnetic field rotating at a frequency of $0.5 \mathrm{~Hz}$ with a magnitude of $30 \mathrm{mT}$. Figure 9 illustrates consecutive images of this motion behavior of the microrobot. Images a) to d) show the intravitreal microrobot at a constant orientation whereas the magnetic field, indicated by a black arrow, changes its angle. When the angle between the magnetic field and the microrobot becomes larger than $90^{\circ}$, the microrobot quickly aligns with the field and, subsequently, starts following the field with a delay until it pauses again. Using a microscope, thin collagen fiber bundles can be observed that are attached to the intravitreal microrobot. Therefore, it is concluded, that the insert is entangled in the elastic fibers, which constrain the microrobot mobility in vitreous.

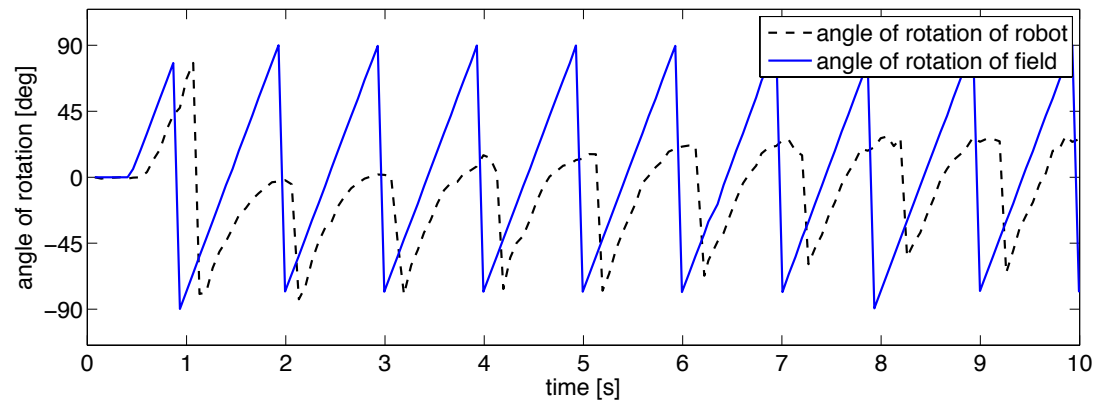

Figure 8: Angle of rotation of the applied magnetic field $(10 \mathrm{mT})$ and the microrobot in lapine vitreous (in vivo) at field rotation frequency $0.5 \mathrm{~Hz}$ 

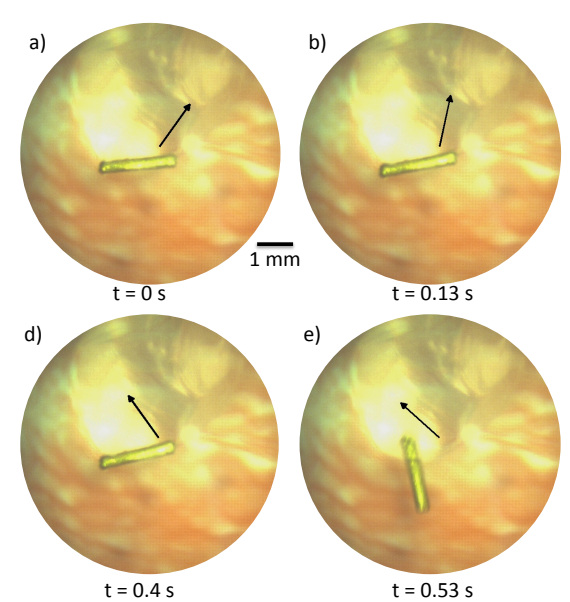

$\mathrm{t}=0.13 \mathrm{~s}$
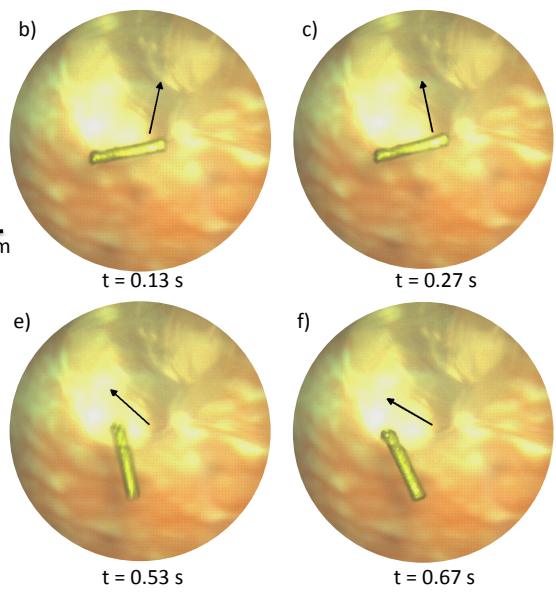

Figure 9: Microrobot in lapine vitreous. In a) to d) the microrobot maintains the same orientation, while the field (indicated by a black arrow) changes orientation. When a large difference is reached $\left(>90^{\circ}\right)$, the robot quickly follows the field e) and starts rotating as normally d).

In order to compare the mobility of an intraocular microrobot in different media, Figure 10 summarizes the correlation between the relative time delay of a microrobot in lapine and porcine vitreous, BSS and silicone oil, and the rotation of the magnetic field. The relative delay, defined by the time delay normalized by the period of rotation, is plotted against the period and its frequency in the surrounding media. The figure illustrates that relative time delay decreases with decreasing frequency and thus, increasing period $\mathrm{T}$.

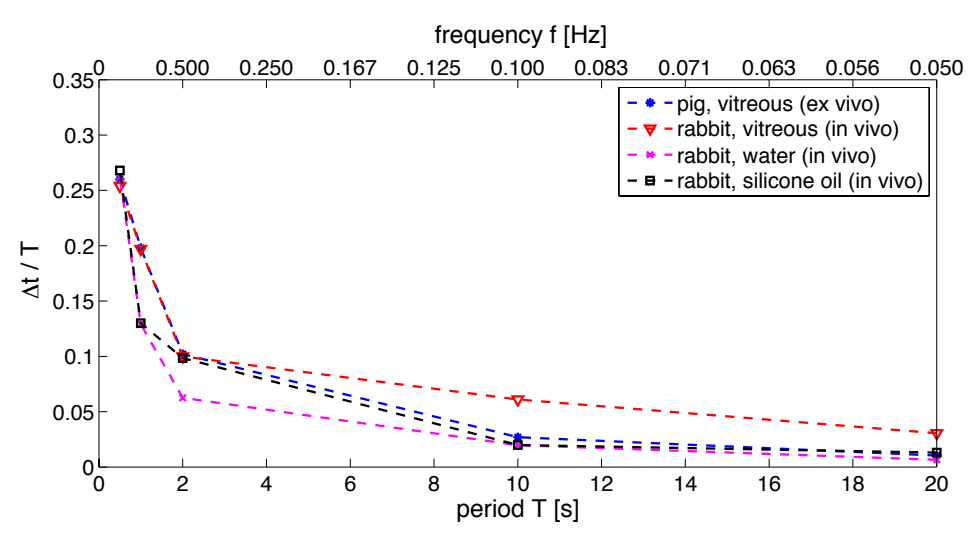

Figure 10: Ratio between the time delay of the microdevice to the field rotation and the period of the field rotation $T$, plotted against the period of the field rotation in seconds.

\subsection{Translational Movement}

The force resulting in the translation of the intraocular microrobot is caused by an applied magnetic field gradient generated by the OctoMag. Figure 11 shows four consecutive images of a wireless microrobot inside the vitrectomized rabbit eye, surrounded by BSS. A magnetic field gradient is applied along the horizontal direction causing the microrobot to translate along this axis. 

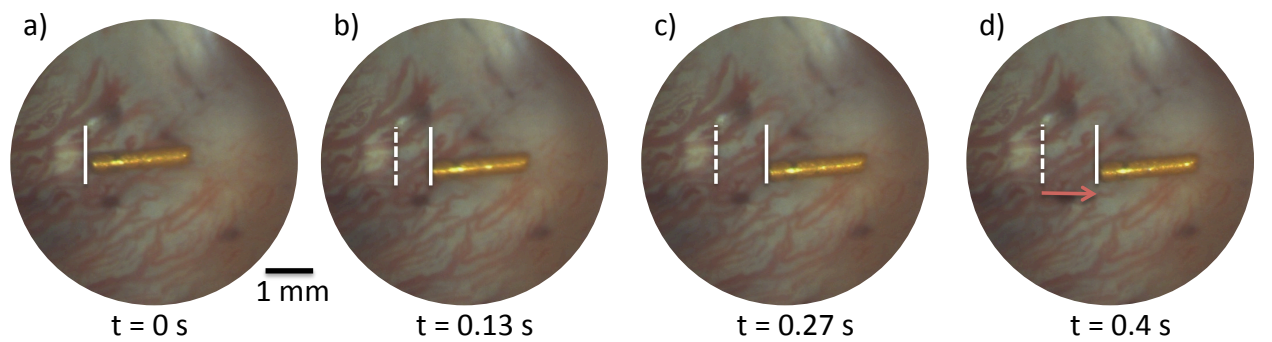

Figure 11: Wireless microrobot inside the vitrectomized (in vivo) rabbit eye surrounded by BSS. A magnetic field gradient is applied causing the microrobot to translate.

Figure 12 illustrates translational movement of a microrobot in lapine vitreous. The red line shows the position of the microrobot as a result of an increasing gradient along the axis of robot orientation at constant field magnitude $(20 \mathrm{mT})$. A general increase of translation due to increasing field gradient is observed. However, the translation of the microrobot in vitreous is less than $0.2 \mathrm{~mm}$ for a magnetic gradient, that increases piecewise from 0 to $500 \mathrm{mT} / \mathrm{m}$ over 20 seconds, as seen in Figure 12. It is assumed that collagen fiber bundles that attach to the microrobot in vitreous cause the lack of translational movement. The interaction between the microrobot and collagen fiber bundles can be modeled as a mass-spring system. Figure 13 shows the response of a microrobot that is entangled in a collagen fiber bundle within lapine vitreous. Magnetic gradients between 0 and $300 \mathrm{mT} / \mathrm{m}$ are applied at a constant field magnitude of $30 \mathrm{mT}$. It is shown that the applied force is proportional to microrobot displacement. Thus, collagen fiber bundles show the behavior of a linear spring.

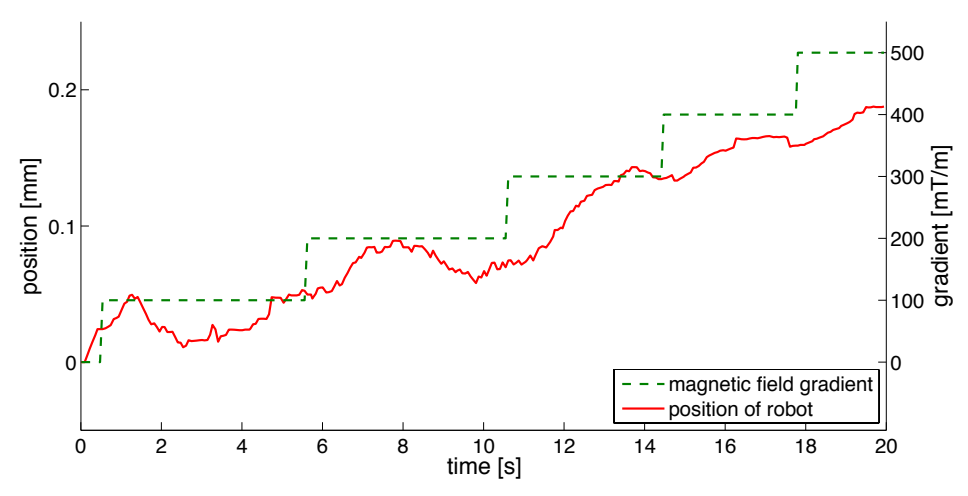

Figure 12: Translation of intravitreal microdevice due to magnetic field gradient at a constant field of $20 \mathrm{mT}$ in lapine vitreous (in vivo) 


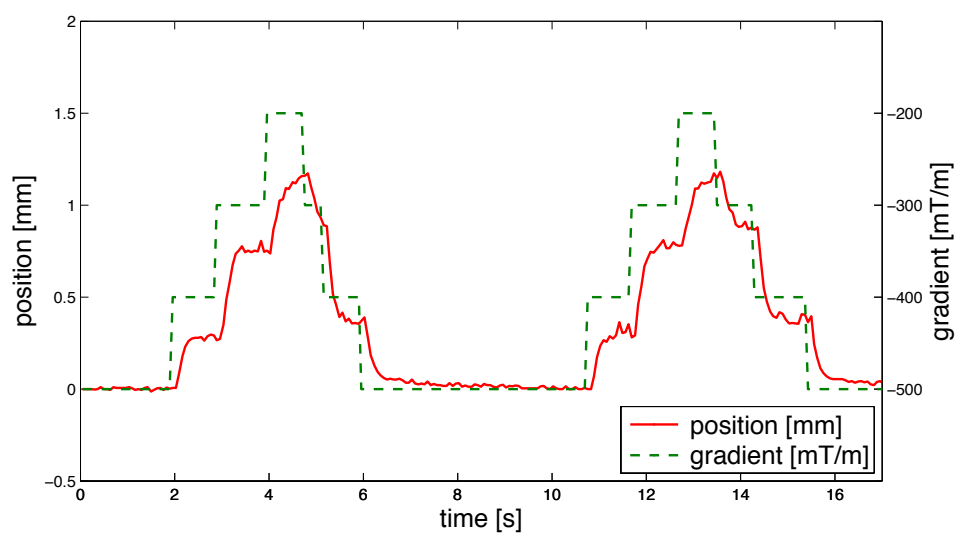

Figure 13: Translational movement of intravitreal microrobot that is entangled in collagen fiber bundle at $30 \mathrm{mT}$. The applied magnetic gradient is directly proportional to the displacement of the microrobot. The microrobot that is attached to a collagen fiber bundle can be modeled as a spring-mass system.

Figure 14 illustrates the behavior of the microrobot in BSS inside the living rabbit eye. The red line shows the position of the microrobot within the eye, whereas the green dashed line indicates the increasing field gradient. Similar to in vivo experiments in vitreous, the general trend shows increasing translation for increasing magnetic field gradients. The translation of the microrobot in BSS is an order of magnitude larger than in lapine vitreous with a maximum of $4 \mathrm{~mm}$. As stated before, BSS is less viscous than vitreous, thus, allowing for better mobility of the microrobot. Furthermore, no collagen fibers are present in BSS in that the microrobot can entangle. However, due to the low viscosity of BSS, the microrobot sinks to the retina instead of floating within the central eye. When applying a magnetic field gradient, the microrobot sticks to the retina until a critical gradient is applied that generates a force, which is large enough to overcome friction. The black arrow in Figure 14 indicates a characteristic stick-slip friction behavior of the microdevice in BSS. In the in vivo experiment the device starts to translate at this critical field gradient $(420 \mathrm{mT} / \mathrm{m})$, as illustrated in the figure.

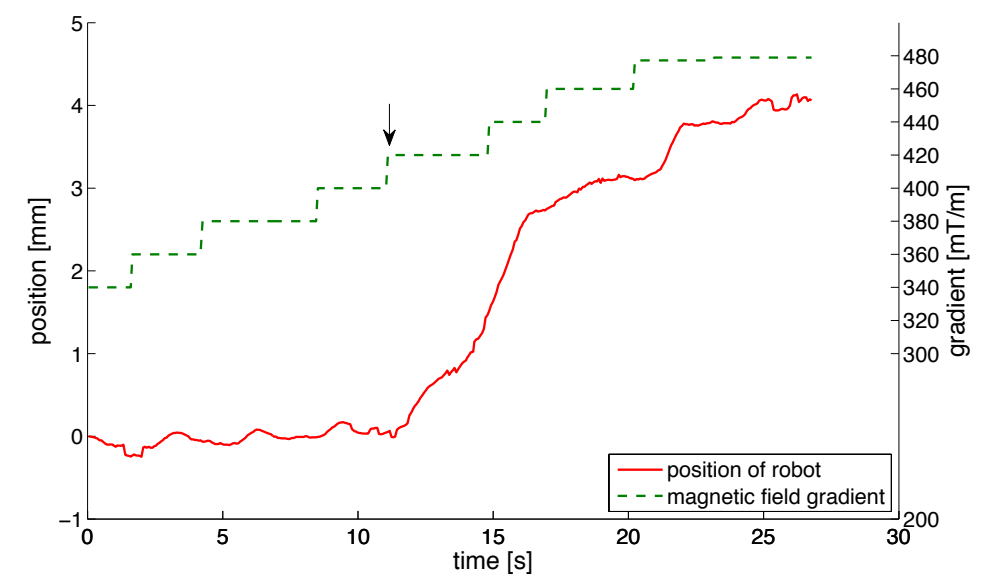

Figure 14: Translation of microdevice due to magnetic field gradient at a constant field of $30 \mathrm{mT}$ in BSS inside the vitrectomized lapine eye (in vivo); the critical gradient is indicated by the black arrow. 
Figure 15 illustrates the microrobot response to various gradients at a constant field magnitude $(20 \mathrm{mT})$ in porcine vitreous. It is observed, that an increasing field gradient results in an increased translation of the microrobot. Furthermore, a typical viscoelastic behavior of the vitreous is observed. By applying a constant gradient, a constant force is exerted on the device. However, the translational movement of the microdevice shows a delayed creep response to the force input. At a field magnitude of $20 \mathrm{mT}$ the maximum translation of the robot is measured to be $3.2 \mathrm{~mm}$ in porcine vitreous. The resulting maximal translational displacements of an intraocular microrobot in porcine vitreous as a result of changing field gradients are summarized in Table 1. The table shows that the translational displacement increases with increasing magnetic gradient, as well as increasing field magnitude. However, the gradient generally has a larger influence on the translational behavior of the intravitreal microdevice.

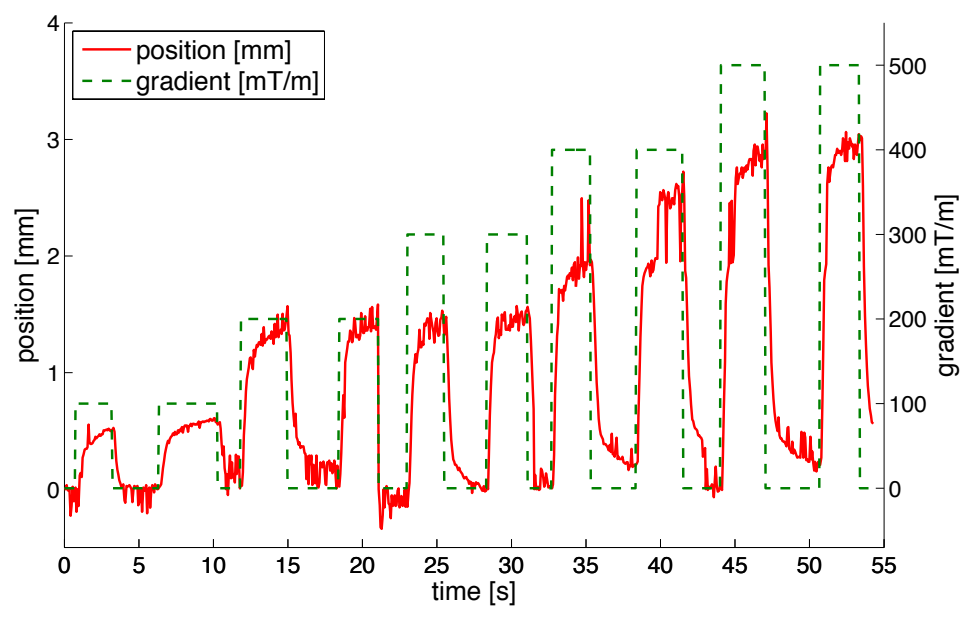

Figure 15: Translation of intravitreal microdevice due to magnetic field gradient at a constant field of $20 \mathrm{mT}$ in porcine vitreous (ex vivo)

Table 1: Maximal translational displacement in $\mathrm{mm}$ due to magnetic gradient $[\mathrm{mT} / \mathrm{m}]$ at constant field magnitude $[\mathrm{mT}]$ in porcine vitreous (ex vivo)

\begin{tabular}{|c|c|c|c|c|c|}
\hline $\begin{array}{l}\text { Gradient } \\
\text { Field mag. }\end{array}$ & $100 \mathrm{mT} / \mathrm{m}$ & $200 \mathrm{mT} / \mathrm{m}$ & $300 \mathrm{mT} / \mathrm{m}$ & $400 \mathrm{mT} / \mathrm{m}$ & $500 \mathrm{mT} / \mathrm{m}$ \\
\hline $10 \mathrm{mT}$ & 0.2 & 0.6 & 1.1 & 1.4 & 1.7 \\
\hline $20 \mathrm{mT}$ & 0.5 & 1.6 & 1.5 & 2.7 & 3.2 \\
\hline $30 \mathrm{mT}$ & 1.1 & 1.5 & 2.4 & 3.0 & 3.0 \\
\hline $40 \mathrm{mT}$ & 1.3 & 2.2 & 2.4 & - & - \\
\hline
\end{tabular}

When comparing results from the in vivo experiments in lapine vitreous with those from ex vivo experiments in porcine vitreous, it is observed that translational displacement of the intravitreal microdevice is approximately one order of magnitude larger in porcine vitreous.

Furthermore, none of the rabbit eyes under observation showed immediate inflammation during the experiments or upon removal of the microrobot. 


\section{Conclusion}

This work demonstrates the general feasibility of controlling the movement of a wireless microdevice inside the eye. As the microrobot can be injected into the eye, steered and removed from the vitreous cavity by a surgeon, this technology potentially offers the possibility to assist in minimally invasive ophthalmic treatments. Future applications include localized slow-release targeted drug delivery and assistance in mechanical operations inside the eye.

Lapine in vivo and porcine ex vivo experiments show that a microdevice can be injected into the vitreous cavity, filled with vitreous, BSS or silicone oil, and moved inside the vitreous cavity. Rotational as well as translational mobility of the microdevice have been explored. It is shown that the microrobot rotation is similar in the four surrounding media investigated, only being dependent on the rotational frequency of the applied magnetic field. Thus, a surgeon using this technology has better control over the intraocular microrobot at low rotation frequencies and low field magnitudes due to decreased instabilities and magnetic drift. Microrobot translation depends on the applied field gradient and the viscosity and elasticity of the surrounding medium. In the experiments, vitreous exhibits a viscoelastic behavior, and the microdevice can get caught in bundles of collagen fibers, which reveal spring like characteristics. The experiments show that the microrobot has increased translational mobility in cadaver porcine vitreous compared to living lapine vitreous. Reasons for this observation are higher density of rabbit vitreous and the post mortem liquefaction ${ }^{27}$ of the vitreous in the cadaver pig eyes.

For future purposes the microrobot can be microfabricated with specific designs. The size as well as the shape of the microrobot can be adjusted to the task. For slow-release targeted drug delivery the microrobot features a drug reservoir, whereas mechanical components, such as needles or hooks, are attached for mechanical applications. The microrobot dimensions are only restricted by the inner diameter of a $23 \mathrm{G}$ needle, which is used for suture-less injection through the pars plana region of the sclera into the eye. The electromagnetic control system, OctoMag, allows for precise control of the microrobot in 3D by generation of an oriented magnetic field and gradient. The risk of ophthalmic surgery can potentially be reduced to a minimum with the assistance of minimally invasive microrobots. Changes in position, which are beyond human perception, can be tracked with high resolution by the surgeon during an operation. Additionally, the forces applied to the robot can be derived from the system inputs. Moreover, the absence of human tremor reduces the risk of damaging the delicate structures in the eye.

Ongoing research focuses on future applications of microrobots inside the eye globe, especially assistance in the treatment of maculopathies, such as AMD, intravenous deployment of anticoagulation agents for RVO and mechanical applications, like epiretinal membrane peeling. Additionally, we focus on minimally invasive removal of a microrobot and tissue material from the 
ocular globe. Furthermore, current research addresses the development of coatings to minimize adhesion between the microrobot and collagen fibers in the vitreous. Further studies on the biocompatibility of intravitreal microrobots are currently ongoing.

To summarize, this work investigates microrobots as a tool for minimally invasive intraocular surgery and demonstrates the mobility of a microrobot inside the living eye, surrounded by vitreous, BSS and silicone oil. Rotational as well as translational mobility of the microrobot have been investigated and the potential of an intravitreal microrobot assisting in surgery inside the eye, such as targeted drug delivery or mechanical applications, is examined. Microrobots can augment the capabilities of ophthalmic surgeons and aid them in achieving safer and more precise interventions with reduced recovery times for the patient. As a result, they can be considered a compelling assistive technology for ophthalmic surgery.

\section{Acknowledgements}

The authors gratefully acknowledge the assistance of Prof. Dr. Bernhard Spiess, head of the ophthalmology department of the Animal Hospital Zurich, for all ophthalmic operations. Furthermore, thanks go to Dr. Simon Pot and Dr. Katja Nuss of the Animal Hospital Zurich.

\section{References}

1. Charles S. Debating the Pros and Cons of 23-g vs. 25-g Vitrectomy. Retinal Physician. 2006;3(1):24 - 25.

2. Taylor R, Jensen P, Whitcomb L, et al. A steady-hand robotic system for microsurgical augmentation. The International Journal of Robotics Research. 1999;18(12):1201 - 1210.

3. Mitchell B, Koo J, Iordachita I, et al. Development and Application of a New Steady-Hand Manipulator for Retinal Surgery. Paper presented at: 2007 IEEE International Conference on Robotics and Automation, 2007; Rome.

4. Bettini A, Marayong P, Lang S, Okamura AM, Hager GD. Vision-assisted control for manipulation using virtual fixtures. IEEE Trans. Robotics. 2004;20(6):953 - 966.

5. Becker BC, Valdivieso CR, Biswas J, Lobes LA, Riviere CN. Active guidance for laser retinal surgery with a handheld instrument. Paper presented at: Int. Conf. Engineering in Medicine and Biology Society, 2009.

6. Wei W, Goldman RE, Fine HF, Chang S, Simaan N. Performance Evaluation for Multi-arm Manipulation of Hollow Suspended Organs. IEEE Transactions on Robotics. 2009;25(1):147 - 157.

7. Eljarrat-Binstock E, Pe'er J, Domb AJ. New Techniques for Drug Delivery to the Posterior Eye Segment. Pharmaceutical Research. 2010;27(4):530 - 543.

8. Davies NM. Biopharmaceutical considerations in topical ocular drug delivery. Clinical and Experimental Pharmacology and Physiology. 2000;27:558 - 562.

9. Michels S, Schmidt-Erfurth U, Rosenfeld PJ. Promising new treatments for neovascular age-related macular degeneration. 2006;15(7):779 - 793.

10. Yasukawa T, Ogura Y, Tabata Y, Kimura H, Wiedemann P, Honda Y. Drug delivery systems for vitreoretinal diseases. Progress in Retinal and Eye Research. 2004;23(3):253 - 281.

11. Choonara YE, Pillay V, Danckwerts MP, Carmichael TR, du Toit LC. A review of implantable intravitreal drug delivery technologies for the treatment of posterior segment eye diseases. Journal of pharmaceutical sciences. 2010;99(5):2219 - 2239.

12. Allergan I. Ozurdex (dexamethasone intravitreal implant). 2012. Available at: http://www.ozurdex.com/AboutOzurdex.aspx. Accessed 2013. 
13. Shahid H, Hossain P, Amoaku WM. The management of retinal vein occlusion: is interventional ophthalmology the way forward? British J. Ophthalmology. 2006;90(5):627 - 639.

14. Gupta PK, Jensen PS, de Juan EJ. Surgical forces and tactile perception during retinal microsurgery. Medical Image Computing and Computer-Assisted Intervention - MICCAI'99. Vol 1679. Cambridge: SpringerVerlag; 1999:1218 - 1225.

15. Jagtap AD, Riviere CN. Applied Force during Vitreoretinal Microsurgery with Handheld Instruments. Paper presented at: Engineering in Medicine and Biology Society, 2004. IEMBS'04. 26th Annual International Conference of the IEEE, 2004.

16. Myles ME, Neumann DM, Hill JM. Recent progress in ocular drug delivery for posterior segment disease: Emphasis on transscleral iontophoresis. Advanced Drug Delivery Reviews. 2005;57:2063 - 2079.

17. Mirshahi A, Roohipoor R, Lashay A, Mohammadi SF, Mansouri MR. Surgical induction of chorioretinal venous anastomosis in ischaemic central retinal vein occlusion: a non-randomised controlled clinical trial. British J. Ophthalmology. 2005;89(1):64 - 69.

18. Bergeles C, Kummer MP, Kratochvil BE, Framme C, Nelson aBJ. Steerable intravitreal inserts for drug delivery: in vitro and ex vivo mobility experiments. Paper presented at: Medical Image Computing and Computer-Assisted Intervention-MICCAI 2011, 2011.

19. Nelson BJ, Kaliakatsos IK, Abbott JJ. Microrobots for minimally invasive medicine. Annual Review of Biomedical Engineering. 2010;12:55 - 85.

20. Vartholomeos P, Fruchard M, Ferreira A, Mavroidis C. MRI-guided nanorobotic systems for therapeutic and diagnostic applications. Annual Review of Biomedical Engineering. 2011;13:157 - 184.

21. Sivaraman KM, Kellenberger C, Pané S, et al. Porous polysulfone coatings for enhanced drug delivery. Biomedical microdevices. 2012;14:603-612.

22. Sivaraman KM, Bayrakceken K, Ergeneman O, et al. Tailoring the drug loading capacity of polypyrrole films for use in intraocular biomicrorobots. Paper presented at: IEEE Int. Conf. Engineering in Medicine and Biology, 2010.

23. Kummer MP, Abbott JJ, Kratochvil BE, Borer R, Sengul A, Nelson BJ. OctoMag: An Electromagnetic System for 5-DOF Wireless Manipulation. IEEE Transactions on Robotics. December 2010;26(6):10061017.

24. Lee B, Litt M, Buchsbaum G. Rheology of the vitreous body. Part I: Viscoelasticity of human vitreous. Biorheology. 1992;29(5-6):521-533.

25. Bishop PN. Structural macromolecules and supramolecular organisation of the vitreous gel. Progress in retinal and eye research. 2000;19(3):323-344.

26. Lee B, Litt M, Buchsbaum G. Rheology of the vitreous body Part II: Viscoelasticity of Bovine and Porcine vitreous. Biorheology. 1994;31(4):327-338.

27. Sebag J. Ageing of the vitreous. Eye. 1987;1(2):254-262.

28. The Federal Authorities of the Swiss Confederation. Tierschutzverordnung (TSchV). June 1, 2012. Available at: http://www.admin.ch/ch/d/sr/455_1/index.html.

29. Sharif-Kashani P, Hubschman JP, Sassoon D, Kavehpour HP. Rheology of the vitreous gel: effects of macromolecule organization on the viscoelastic properties. Journal of Biomechanics. 2011;44(3):419-423. 